\title{
Empty Sea
}

Michael Voulgarellis

If the sea was empty

Could we pick daisies off the ocean floor?

Would the fish sprout legs

And stroll across the non-existent shore?

Would we be left instead

To swim across the skies?

And how would a poor poet describe

His affection to blue eyes?

We'd all be left

To drown in oxygen

Tell the children stories

Of way back when

The sea she roared

Ripping apart the shore

But now the sea

She is no more 